\title{
Workplace Based Assessment Program for International Medical Graduates: An Evaluation of an Australian Trial Site
}

\author{
Daniel Terry", Blake Peck, Swapnali Gazula \\ School of Health, Federation University Australia, Ballarat, 3350 Victoria, Australia
}

Received July 30, 2020; Revised October 26, 2020; Accepted November 1, 2020

\section{Cite This Paper in the following Citation Styles}

(a): [1] Daniel Terry, Blake Peck, Swapnali Gazula, "Workplace Based Assessment Program for International Medical Graduates: An Evaluation of an Australian Trial Site," Universal Journal of Public Health, Vol. 8, No. 6, pp. 198 - 206, 2020. DOI: 10.13189/ujph.2020.080603.

(b): Daniel Terry, Blake Peck, Swapnali Gazula (2020). Workplace Based Assessment Program for International Medical Graduates: An Evaluation of an Australian Trial Site. Universal Journal of Public Health, 8(6), 198 - 206. DOI: 10.13189/ujph.2020.080603.

Copyright $\bigcirc 2020$ by authors, all rights reserved. Authors agree that this article remains permanently open access under the terms of the Creative Commons Attribution License 4.0 International License

\begin{abstract}
There is a need to understand Workplace Based Assessment programs in Australia to improve future offerings. This paper evaluates the efficacy of a Workplace Based Assessment program at a single regional Australian healthcare location using a mixed-method approach to collect data from three sources. Tools included Mini-Clinical Evaluation Exercise, case-based discussion and in-training assessment, and structured face-to-face interviews with eighteen key-stakeholders. The Workplace Based Assessment program itself was evaluated against a number of indicators, including the psychometric properties assessed by each of the tools used. In addition, the adequacy of the current program and specifically the tools used within it to determine the clinical competence and safety of International Medical Graduates was examined. Lastly, satisfaction of key-stakeholders with program preparation and usability of tools was investigated. The results indicate that the tools currently used within the Workplace Based Assessment program continue to be reliable, and assessors are assessing International Medical Graduates with a high degree of consistency across the program. In addition, this study found that both International Medical Graduates and assessors remain satisfied with regard to confidence, usability and user perception of the current Workplace Based Assessment methods to provide a more meaningful experience for those being assessed. Overall, this evaluation provides insights into the current Workplace Based Assessment program and
\end{abstract}

makes recommendations for future improvements. These include clearer program guidelines; greater opportunities for support of International Medical Graduates, feedback for and further training of assessors.

Keywords Healthcare, Workplace Based Assessment, International Medical Graduates, Training, Registration

\section{Introduction}

Although Australia's medical workforce is growing, its growth is not proportionate in clinical specialties, geographical distribution, and ageing population within the country [1]. There is a predicted shortage of medical doctors in Australia, with a steep decline predicted in 2025 [1]. International Medical Graduates (IMGs), also known as Overseas Trained Doctors (OTDs) are considered one of the solutions to quickly respond to this deficit. In Australia, IMGs currently contribute to approximately a quarter of the overall general practice registrars and many other medical roles across the country [2]. In terms of addressing the current shortages, IMGs are relied on to address this gap and IMGs will continue to be central in health workforce planning across Australia in the coming years [1, 3-7].

The Australian Medical Council (AMC) offers a number of pathways to assess suitability for registration of IMGs 
for working as a medical practitioner in Australia [8-11]. In most cases an AMC certificate of registration is issued after rigorously verifying an IMG's credentials; scrutinising the examination and accreditation processes of the applicant's country of origin; and the administration of a two part AMC certification process including a Multiple Choice Question (MCQ) exam [12] followed by the AMC clinical examination [13].

In addition to the standard process, IMGs can also seek registration through the 'alternate' Standard Pathway, which involves the Workplace-Based Assessment (WBA) pathway. In this alternate pathway, instead of completing a clinical skills examination, an IMG is assessed for up to twelve months in terms of contextualised 'performance' in a clinical setting that encompasses a breadth of clinical possibilities rather than a de-contextualised examination of their 'competence' alone [12]. Thus, the WBA emphasises the assessment of a medical practitioner's actual performance in practice [13-16].

The WBA program was initially developed in 2010 by medical academics from New South Wales, Australia. It was subsequently accredited by the Australian Medical Association (AMA), in the same year, as an alternative to the usual pathway to assess an IMGs suitability for registration [17]. One of the reasons for seeking alternative registration pathways was the increased incidence of complaints and adverse disciplinary actions against IMGs by the AMA as compared to the Australian trained doctors. One of the reasons cited for this was the disparity in the professional learning and experience of IMGs from various countries, where education and practices have a propensity to differ. The lack of Australian context further created a huge challenge for IMGs in quickly adapting to work within Australia. The WBA was initiated to bridge this gap by providing a contextualised experience in assessing IMGs through opportunities to interact with patients and staff. While there is no training offered within the WBA, learning opportunities are created through regular feedback and self-reflections. Furthermore, with successful completion of the WBA, IMGs are often recruited in the same health facility, thereby reducing recruitment costs [17]. However, despite offering positive prospects to the institutions, medical workforce, and the IMGs, limited WBA capacity presents an ongoing challenge in Australia, where uptake of the program is dependent on factors such as state or health services need or desire to use the program and the health service's capacity to be accredited to use the program [18].

In order to implement the WBA program, health service providers need to meet certain assessment requirements outlined by the AMC, with room to customise the program in terms of a suite of additional assessment tools. This approach provides the basis to assess the various aspects of the IMGs performance over time [19, 20]. The assessments may include a range of both direct and indirect observation methods at differing times within the program and may include the Mini-Clinical Evaluation Exercise (Mini-CEX), day-to-day direct supervision, direct observation of procedural skills (DOPS), in-training assessment (ITA), case-based discussion (CBD), multi-source feedback (MSF) or $360^{\circ}$ feedback, simulation exercises, an assessment of a portfolio of work, reflective practice, and log books [19]. The AMC has indicated these assessment tools are standardized, well validated, and commonly used, given they provide accurate judgments regarding an IMG's capacity to practice. Furthermore, using of a combination of these direct and indirect observation tools constitute an effective assessment within the WBA program [19].

Although not an exhaustive list, the Mini-CEX, CBD and ITA remain commonly used assessment strategies, which allows a level of flexibility within the WBA program to meet the needs of the IMGs at the hospital level, however the AMC is continuously improving the WBA program to ensure the training program is further standardised.[19] The Mini-CEX and CBD have been used and studied over a number of years to consistently have a moderate to high feasibility and satisfaction levels with high completion rates, as well as strong internal consistency, reproducibility, and construct validity [21-25]. The Mini-CEX is a work based assessment tool that involves the direct observation of a trainee in a focused clinical environment. It is immediately followed by feedback aimed to guide trainees learning and improve clinical performance. There are seven questions included in the feedback, using a nine-point Likert scale for assessment as well as a series of free-text feedback opportunities [26]. The CBD tool is also a work-based tool used to evaluate professional judgement in clinical cases, where an assessor selects a case that the trainee has had a significant role in the decision-making process. The structured CBD tool is then used to assess the clinician's clinical judgement and capacity using, similar to the Mini-CEX, a seven question nine-point Likert scale for the assessment in addition to free-text feedback opportunities [27].

The third tool, the ITA (or supervisor report), is based upon first-hand observation by a variety of assigned health-care professionals, such as nurses, pharmacists and other health care personnel who can comment on various aspects of the IMG. These aspects include ethical behaviour, communication, inter-personnel skills, reliability and professional integrity [19]. Although reliability of ITA remains a concern due to the potential for individual subjectivity, when used by a number of personnel in assessing an IMG, its reliability as a tool strengthens. It also provides vital feedback from a diverse number of sources which inform practice. The ITA is a tool to compliment the CBD and Mini-CEX, which all seek to track an IMG's progress. The ITA consists of a formative and summative process that required a supervisor to assess an IMG on 11 clinical items at least twice in any given clinical rotation. ITAs occur particularly at the mid-point and end of clinical rotations, with at least four ITAs being completed over the life of the WBA assessment period [28, 29]. 
Although the WBA is an alternative pathway, a 2012 Australian Government inquiry into registration processes and the provision of support offered to IMGs, showed the WBA was currently not endorsed by all Australian jurisdictions and remains a limited approach to IMG registration in Australia [12]. In fact, the WBA is limited to ten national sites, including limited, yet specific, health services in New South Wales, South Australia, Queensland, Victoria, Western Australia, and Tasmania [12]. This highlights the need to understand WBA programs to improve future offerings. The following case study presents evaluation of one such WBA program at an Australian location, and provides suggestions for future programs.

At the time of the Government inquiry, there were concerns regarding the limited availability of WBA places and associated questions regarding the quality and independence of the WBA review [12]. In addition, there have been concerns raised about the fiscal implications for the government and wider public of such programs; however, whether such an investment represents value for money remains to be seen among the individual health services who are AMC accredited to use the alternate Standard Pathway - WBA program [26].

\section{Aim and objectives}

Considering the concerns from the Government inquiry [12] regarding the quality and independence of the WBA review, a call was made for an evaluation of the WBA at an Australian health service which was utilising the program. Therefore, the aim of this study was to examine the efficacy of the WBA program within a given context. As such, the objectives of the evaluation study were to specifically examine the reliability of the assessment tools, the constancy of scores between assessors, while also understanding the utility of the program among assessors and IMGs themselves.

\section{Methods}

The project was an evaluative study using a mixed methods design. A mixed method design is a process where both quantitative and qualitative data are collected in a single study to examine complex or lesser known questions [30]. Both data types are analysed and integrated in the research process for the express purpose of gaining a more comprehensive understanding of the research problem [31]. The rationale for using mixed methods in the current study was to enable the acquisition of an in-depth understanding of WBA in the given context, which was necessary to adequately address the aims of the project: to evaluate the WBA program and make recommendations for change.

\subsection{Study Site}

This study involved an Australian acute care public hospital with capacity of over 300 beds that serviced a regional population of more than 100,000 people. This health service treated over 25,000 inpatients and more than 250,000 outpatients annually. At the time of data collection, the health service had assisted more than 13 IMGs to complete the WBA program with a further group of 8 IMGs who were in the process of completing the program. Three WBA tools were used at the selected health service included the Mini Clinical Evaluation Exercise (Mini-CEX), the Case Based Discussions (CBD) and an IMG In- Training Assessment (ITA), as outlined previously.

\subsection{Data Collection}

To achieve the research aims, data were collected from three sources through a retrospective review of existing paper-based Mini-CEX; CBD as well as ITA IMGs assessments that had been conducted by clinicians who received training and approval to be assessors. This represented all data throughout the program (370 IMG assessment records), which were then individually coded, and each element of the individual assessment were entered into Microsoft Excel and SPSS v24.0 in preparation for analysis. Sample size calculation was not conducted, given all data were used and analysed within the study.

In addition, data were also collected through short (15-20 minutes), structured face-to-face interviews with purposive number of IMGs $(n=4)$ and assessors $(n=14)$. Interview questions pertained to the efficacy of the program, and included questions relating to the benefits and challenges of participating in the program as either an IMG or an assessor, along with areas for future improvement. It must be noted of the eight IMGs that were undertaking the program at the time were all contacted to participate, however, only one responded given the busy schedules, limited time, or having only recently commenced the program. Of the 13 who had completed the program three responded as they were either still working at the hospital or had recently completed the program. The low participant numbers limited the data saturation of the IMG data; however, saturation was achieved among the assessors. Importantly, we adopt the position of other qualitative researchers in regard to data saturation and suggest that as there are always new theoretical inisghts avalable as long as data continues to be collected that data saturation is not genuinly possible [32]. Data were audio recorded with the participant's consent.

\subsection{Data Analysis}

The Mini-CEX, CBD, and ITA data were analysed using SPSS v24.0. Descriptive and interferential analysis was undertaken and included non-parametric tests such as Chi-square tests (Fisher's Exact Test) and inter-rater reliability to examine the association between different aspects of clinical assessments, problem complexity and 
WBA tool adequacy. Results were considered statistically significant at $\mathrm{p}<0.05$.

The interview data were transcribed verbatim into Microsoft Word and thematically analysed, using NVivo v10.0 [33]. Thematic analysis is a method used to systematically identify recurring themes, patterns of living, behaviour and experience which then become a description of the phenomenon [34]. Once developed these descriptions provided the basis for identifying further emergent insights from the data which became the major themes considered representative of the experiences of both assessor and IMGs. Two investigators independently read the transcripts and double checked the themes to ensure trustworthiness of the data analysis [35]. Member checking was undertaken with each participatnt being provided with a copy of their inteview transcript to ensure clarity, validation and accuracy of interview data. This was also undertaken to ensure any highly sensative information was not included within the data.

\subsection{Ethical Considerations}

The study received ethical approval from the Institutional Human Research Ethics Committee prior to be commencing the study (H0012831). Each IMG and Assessor involved within the WBA program were initially mailed an invitation letter, plain language information statement, and consent form. Afterwards, each potential participant was followed up with a phone call to ascertain their interest in the study and to arrange a date and time for interview. Informed consent was obtained from all participants. It must be noted that due to the small sample size and the potential for ease of identification of each IMG, country of origin and specific details concerning prior training, medical background, and current specialties were not collected as part of the Mini-CEX, CBD, and ITA data. Although the cultural background of the participants was noted within the IMGs interview data, this remains undisclosed to maintain anonymity of each IMG participant.

\section{Results}

The analysis of the assessment records demonstrated there were 13 IMGs who had completed the WBA program, while an additional eight were still undertaking and had partially completed the program at the time of the study. Each IMG participated in two clinical rotations while undertaking the six to twelve-month WBA program, and had or were working in the specialty areas of medicine, surgery, emergency medicine, paediatric medicine, psychiatry as well as obstetrics and gynaecology. IMG Assessments were undertaken by a total of 91 different assessors over the life of the program, all of whom had undertaken clinical supervisor training and annualised updates relating to the WBA program. Each principal assessor was either a consultant physician or senior registrar with whom the IMG worked closely with for a specific rotation.

\subsection{Assessment Tool Evaluation}

International Medical Graduates were assessed by the assessors on 370 separate occasions with 197 Mini-CEXs, 102 CBDs and 71 ITAs being conducted. Within the WBA, each IMG was required to pass 22 assessments, which encompassed completing 12 Mini-CEX, six CBD, and four ITA. Assessments were not randomly assigned, but followed a timetable developed by the Workplace Based Assessment Project manager under the direction of the Director of Clinical Training at the health service [16].

The CBD and Mini-CEX clinical assessments involving a broad range of 'patient-specific problems' were selected as cases for the assessment of IMGs. Cases included diverse medical diagnosis such as pneumonia, abdominal pain, acute appendicitis, atrial fibrillation, diarrhoea, cancer, chronic cardiac failure, fracture neck of femur, pancreatitis, post-partum sepsis, snake bites, and falls for investigation.

\subsubsection{Case Based Discussion}

The mean number of CBDs completed by each assessor was 1.1 (range, 0-10). Assessors self-rated the complexity of the clinical problems used within the CBD assessments as being of low complexity (12.7\%), average complexity $(63.7 \%)$ and high complexity $(23.6 \%)$. Conversely, the mean number of Mini-CEXs completed by assessors was 2.2 assessments (range, 0-13) (see Table 1).

Within the CBD, IMGs were assessed and scored against seven items, which include history taking, clinical reasoning, assessment, investigation and treatment, follow-up and management, record keeping, and overall clinical care [16]. The data indicated that overall CBD score among the IMGs was between 'unsatisfactory' to 'superior' (2 to 9 out of 9), with mean scores ranging from $6.16-6.58$ (satisfactory). There was only one occasion identified from the data where an IMG failed a CBD assessment and this IMG was provided with extensive feedback by way of the documentation and subsequently passed an additional CBD with another specialist on a separate occasion, achieving a positive result.

At the completion of the CBD, the assessment was rated by each assessor and a judgment made as to the degree the clinical case was an adequate test of the doctor's abilities. It was shown that $97.1 \%$ of the CBD assessors rated the clinical case as an 'adequate' to 'superior' test of the IMGs abilities.

The reliability and internal consistency of the CBD was calculated to demonstrate if various questions from each assessment were consistently and reliably measuring the same thing. Cronbach Alpha $(\alpha)$ was used to assess the reliability of the tool, where a coefficient above .7 is 
considered acceptable and scores above .8 are preferable for internal consistency [36]. A high-level internal consistency was found for the CBD $(\alpha=.894, \mathrm{n}=102)$.

Lastly, the inter-rater reliability of the CBD assessment scores was determined between the assessors by using the intra-class correlation coefficient (ICC). This was used to demonstrate if all assessors are reliably or consistently assessing IMGs in the same manner. As such, the strong inter-rater reliability scores, between assessors for CBD, $\mathrm{F}(2,612)=19.339, \mathrm{p}=.000$, produced under the two-way random effects model, showed that assessors were significantly reliable when assessing IMGs using the CBD (see Table 2). This suggests that no matter who conducted the CBD assessment, IMGs were being assessed in a similar way.

\subsubsection{Mini-CEX}

The average Mini-CEX duration was 23.6 minutes (range, 10-60 minutes), while the average time for feedback was 13.2 minutes (range, 3-40 minutes). Assessors also self-rated the clinical complexity of the cases identified for the Mini-CEX assessments as being of low complexity $(11.1 \%)$, average complexity $(70.0 \%)$ and high complexity (18.9\%) (see Table 1).

In terms of the seven Mini-CEX clinical items which IMGs were assessed and scored against, results ranged from 'satisfactory' to 'superior' (5 to 9 out of 9), with the mean scores ranging from $6.62-7.24$ (satisfactory to superior). There were three instances of IMGs having been identified as 'did not meet expectations' within an overall Mini-CEX assessment and those IMGs who had not met expectations were subsequently provided with feedback, and went on to complete and pass an additional Mini-CEX assessment with other senior specialists.

At the completion of the Mini-CEX, the assessment itself was rated by each assessor and a judgement made as to the degree the clinical case was an adequate test of the doctor's abilities. It was shown that $97.0 \%$ of the Mini-CEX assessors rated the clinical case as an 'adequate' to 'superior' test of the IMGs abilities.
The reliability and internal consistency of each of the mini-CEX was calculated to demonstrate if various questions from each assessment were consistently and reliably measuring the same thing. Cronbach Alpha $(\alpha)$ was again used and demonstrated a high-level of internal consistency for the Mini-CEX ( $\alpha=.964, \mathrm{n}=197)$.

Lastly, the inter-rater reliability of the Mini-CEX scores was determined between the assessors by using the ICC. Again, this was used to demonstrate if all assessors are reliably or consistently assessing IMGs in the same manner. The strong inter-rater reliability scores, between assessors for Mini-CEX, $F(6,1170)=17.234, p=.000$, produced under the two-way random effects model, showed that assessors were significantly reliable when assessing IMGs using the Mini-CEX (see Table 2), again suggesting that no matter who conducted the Mini-CEX, IMGs were being assessed in a similar way.

\subsubsection{In-Training Assessment}

It must be noted that of the 11 clinical items assessed in the ITA fell between 'consistent with level of appointment' and 'performance better than expected' (3 to 4 out of 6), with the mean scores ranging from $3.77-4.22$. No IMGs had failed an ITA. The reliability and internal consistency of each of the ITA was calculated to demonstrate if various questions from each assessment were consistently and reliably measuring the same thing. Cronbach Alpha $(\alpha)$ was used again and demonstrated a high-level of internal consistency for the ITA $(\alpha=.926, \mathrm{n}=71)$.

Again, the inter-rater reliability of the ITA scores was determined between the assessors by using the ICC to demonstrate if all assessors are reliably or consistently assessing IMGs in the same manner. As such, the strong inter-rater reliability scores, between assessors for ITA, $\mathrm{F}(2,630)=3.841, \mathrm{p}=.000$, produced under the two-way random effects model, showed that assessors were also significantly reliable when assessing IMGs using the ITA (see Table 2).

Table 1. Level of complexity of the case being assessed

\begin{tabular}{ccccc}
\hline Assessment method & $\begin{array}{c}\text { Mean number } \\
\text { completed by assessors }\end{array}$ & $\begin{array}{c}\text { Low } \\
\text { Complexity }\end{array}$ & $\begin{array}{c}\text { Average } \\
\text { Complexity }\end{array}$ & $\begin{array}{c}\text { High } \\
\text { Complexity }\end{array}$ \\
\hline CBD & 1.1 (range, 0-10) & $13(12.7 \%)$ & $65(63.7 \%)$ & $24(23.6 \%)$ \\
Mini CEX & $2.2($ range, 0-13) & $22(11.1 \%)$ & $138(70.0 \%)$ & $37(18.9 \%)$ \\
\hline
\end{tabular}

Table 2. Inter-rater reliability of assessors in WBA program

\begin{tabular}{ccccc}
\hline Assessment method & Number of assessments & ICC & Degrees of freedom (df) & Confidence interval (CI) \\
\hline CBD & 102 & .948 & 2,612 & $.875-.989$ \\
Mini CEX & 197 & .942 & 6,1170 & $.860-.988$ \\
ITA & 71 & .740 & 2,630 & $444-.922$ \\
\hline
\end{tabular}




\subsection{Assessor and IMG Perspectives Regarding the WBA Program}

In addition to the retrospective evaluation of completed assessment tools, interviews were conducted with four IMGs, out of which three had completed the WBA program. Additionally, 14 assessors who had between them undertaken a third of the total number of WBA assessments were interviewed. These included 11 specialists, two general practitioners and one visiting medical officer. The following represents a de-identified qualitative thematisation of the experiences of both assessors and IMGs who engaged with the WBA program.

The WBA program was seen as a recruitment drawcard to attract IMGs to the health service and assessors indicated that they had witnessed a higher calibre of IMG working at the health service since the commencement of the program. Assessors reported that the program provided opportunities for gaining useful insights into each IMG; their clinical competence; and their ability to communicate and interact with patients and other health professionals. Assessors and IMGs both felt that the program reduced anxiety, stress and helped new IMGs better understand the Australian medical system.

[IMGs] don't get anxious like in an exam, so they are performing at a level that they would day to day at their work. It also allows you to assess their English skills, presentation skills, relationship to patient skills... so it is a very good way of demonstrating their competencies. (Assessor 14)

The WBA program was observed, by both IMGs and assessors, to be more than an assessment. It was rather a learning process guided by the feedback of assessors. It was a more meaningful experience that provided opportunities for IMGs to learn and develop their skills. There was a high level of confidence and satisfaction with the program including the current tools being used. The program was observed to be a more superior method of assessing the IMGs abilities and skills in real life situations, while ensuring that they were practicing safely in the workplace.

The interaction with the patient is so much more important. You can certainly have a lot of candidates who can sit down and talk about a whole lot of theoretical aspects of cases... [It is about] applying the theoretical framework to a practice situation. (Assessor 11)

Many of those interviewed discussed the subjectivity of the program. This was, in part, due to IMGs being known to the assessors and having worked together. However, rather than an impediment, IMGs saw this familiarity as a positive aspect of the program that helped reduce anxiety, improve communication and skill development. However, concerns were expressed regarding the level at which IMGs were to be assessed, as it was stated some assessors expected IMGs to function at the level of a registrar rather than at the intern level, outlined by the AMC [16]. One IMG said, "some consultants forget ... and they ask the questions as the registrar level" (IMG 3), while another IMG stated they always prepared themselves to answer registrar type questions to ensure they would not be caught out.

Assessors felt that the current range of assessments reasonably represented the seven competencies identified by the AMC as required for registration as a doctor [16]. They felt the current assessment tools were easy to read, simple to complete and acted as a prompt within the assessment process. Nevertheless, there were some concerns regarding the wording of assessment tool when used in areas such as psychiatry, where the assessment "does not always fit the [clinical] wording" (Assessor 7).

In addition, there was little enthusiasm for the adoption of tools such as the Multi-Source Feedback $\left(360^{\circ}\right.$ assessments) and Direct Observation of Procedural Skills (DOPS). Assessors felt their time was limited to use other tools comprehensively, and this was made clear when assessors stated "I don't know if I have got time to do [a $360^{\circ}$ or a DOPs] with these fellows" (Assessor 1), and "I don't know if there is any other [assessment] better really?" (Assessor 2). Despite these concerns, comments were made which suggest support for the collection of data similar to the $360^{\circ}$, which was a current practice when assessing interns.

We actually go and ask the other registrars and other consultants what you think about this person? ... sometimes the nurses [tell us] this intern does this and that ... so we have [the information]. (IMG 1)

\subsection{Program Improvements}

At the conclusion of each interview, participants were asked what improvements could be made to the WBA program to make it more effective. From these interviews, a number of key suggestions were provided. These included clearer program guidelines for both assessors and IMGs regarding the roles of each individual in terms of responsibility for driving the assessment as well as the type of clinical cases that are used; IMGs to be assessed whilst undertaking or immediately after a rotation in a specialist clinical area such as psychiatry; assessors to be used more frequently to maintain their skills; greater opportunities for fostering collegial interaction to build a relationship between IMG and assessor, collaboration and support between assessor in terms of knowing IMGs requirements and ongoing needs; and providing assessors with ongoing follow-up and opportunities for collaboration with their fellow assessors as a means of further training to reduce common errors to ensure greater uniformity and improve overall performance in the program.

\section{Discussion and Conclusions}

International Medical Graduates continue to be central 
to health workforce planning particularly in Australia. A national process is in place to assess IMGs who are seeking to practise medicine in Australia. The accreditation process has a number of alternate pathways, of which one is the 'Standard Pathway - Workplace-Based Assessment'. This is an alternate method to the current Standard Pathway Clinical Examination and is currently being undertaken at ten health services in Australia.

A retrospective analysis of recorded data, which included the records of assessments using the Mini-CEX; CBD and ITA tools, was performed. In addition, an investigation of assessor and IMGs perceptions into current WBA program at one of the key health services was also undertaken to evaluate and make recommendations for the future. This study identified a high level of consistency in the way that assessors are evaluating IMGs across the program being conducted at the study site using a multiple tool approach that supports considerations of the reliability of the current tools in use [13]. Despite research to suggest a lack of evidence to underpin the use of WBAs in medical training [37-39], this study echoes the findings of an Australian systematic review [13] that underscores a high level of confidence from both IMGs and assessors in terms of the satisfaction, usability and perception of the current methods and tools.

Despite the established nature of the approach to IMG assessments, a number of recommendations are made to augment as well as improve the current and future WBA programs. These included clearer program guidelines for both assessors and IMGs, with a particular emphasis on an established timeline for all parties in advance of the assessment. While previous research has not provided clear strategies for addressing logistical issues, there is evidence of identified difficulties in arranging the assessments [40-42].

The provision of opportunities for specialist clinical experience to medical students prior to their assessment of competence in that area has been recognised as standard practice [43]. Considerations to the timing of WBAs with regard to an IMGs exposure to a clinical specialty area have not been identified within contemporary literature. This study recognised that assessors had a desire for students to be currently engaged in or having just completed a clinical specialisation as a trigger for engaging in a WBA for that area, for example, psychiatry.

The remaining recommendations involve the development and/or refinement of attributes in the assessors themselves. The consultants in the current study demonstrated a deep level of engagement with the WBAs of IMGs which is contrary to other larger studies $[40,44]$ with non-IMGs. Despite this, assessors in this study indicated that they would welcome further training in the application of WBAs; a position supported by existing research [44]. This study was able to unpack some of the nuances of what the assessors wanted specifically in a training program. It was identified that having formalised opportunities for various assessors to intermingle might provide a community of practice where it is possible to share and learn how from more experienced clinicians to work with the IMGs within the WBA program. Participant assessors in this study articulated a desire for greater levels of feedback from peers and it could be suggested that peer assessment of the assessor becomes a mandatory component of assessor training in the future.

One limitation identified within this study is the low number of IMGs who agreed to be interviewed with only four (19.0\%) IMGs contributing, resulting in lack of data saturation of the IMG perspectives. The depth of understanding that is achieved through qualitative approaches mitigates the need for large numbers, however we acknowledge that the experiences of IMGs are richly diverse and more interviews with them may well have provided greater insights. Further research is warranted with larger sample size and comparing different pathways for IMG registration. Another limitation to this study was that IMG characteristics such as their country of origin and cultural backgrounds were not disclosed to protect the identity of the small sample. However, these attributes need to be considered in future studies to understand holistic needs of IMGs, which could be incorporated in future WBA programs. This approach will help assessors better support IMGs from heterogenous educational and cultural backgrounds to ease into the Australian Healthcare System with optimal outcomes. Nonetheless, this study provides a basis to understand the WBA program in a regional Australian context. Follow up studies with the IMGs will enable the capture of a wider perspective of their retention and success within the Australian Health Care System.

\section{Declaration of Interest Statement}

No conflict of interest.

\section{Disclosure Statement}

This research did not receive any specific grant from funding agencies in the public, commercial, or not-for-profit sectors.

\section{REFERENCES}

[1] Health Workforce Australia. Australia's future health workforce: Doctors. Canberra: Department of Health; 2014.

[2] Louise M, Tim U. International medical graduates: challenges faced in the Australian training program. Australian Family Physician. 2008;37(6):481-4.

[3] Hawthorne L. International Medical Migration-What Is the 
Future for Australia? MJA Open. 2012;1:18-21.

[4] Spike NA. International Medical Graduates: The Australian perspective. Acad Med. 2006;81(9):842.

[5] Han GS. International medical graduates in Australian news: A media narrative analysis. Journal of Health Organization and Management. 2010;24(3):237-57.

[6] Alexander C, Fraser J. Education, training and support needs of Australian trained doctors and international medical graduates in rural Australia: A case of special needs. Rural Remote Health. 2007;7(681).

[7] Iredale R. Luring Overseas Trained Doctors to Australia: Issues of training, regulating and trading. Int Migr. 2009;47(4):31-65.

[8] Australian Medical Council. Overview of assessment pathways Canberra: Australian Medical Council; 2018 [updated Available from: https://www.amc.org.au/assessme nt/pathways/overview/.

[9] McLean R, Bennett J. Nationally consistent assessment of international medical graduates. Med $\mathrm{J}$ Australia. 2008;188(8):464.

[10] Medical Board of Australia. IMG update. Canberra: Medical Board of Australia; 2010 October.

[11] Australian Medical Council. Registration Issues Paper for the Medical Board of Australia. Canberra: Australian Medical Council; 2009.

[12] House of Representatives Standing Committee on Health and Ageing. Lost in the Labyrinth: Report on the inquiry into registration processes and support for overseas trained doctors. In: House of Representatives SCoHaA, editor. Canberra: Department of House of Representatives Printing and Publishing Office; 2012.

[13] Nair BR, Hensley MJ, Parvathy MS, Lloyd DM, Murphy B, Ingham $\mathrm{K}$, et al. A systematic approach to workplace-based assessment for International Medical Graduates. Med J Australia. 2012;196(6):399.

[14] Office of Postgraduate Medical Education. Review of work-based assessment methods. . Sydney, NSW, Australia: University of Sydney; 2008.

[15] Al-Wardy NM. Assessment methods in undergraduate medical education. Sultan Qaboos University Medical Journal. 2010;10(2):203-9.

[16] Australian Medical Council. Workplace based assessment (Standard Pathway) Kingston, ACT: Australian Medical Council; 2018 [Available from: https://www.amc.org.au/ass essment/pathways/standard-pathway/workplace-based-asse ssment-standard-pathway/.

[17] Nair BR, Searles AM, Ling RI, Wein J, Ingham K. Workplace-based assessment for international medical graduates: at what cost? Medical Journal of Australia. 2014;200(1):41-4.

[18] Singer AH. The potential of workplace - based assessment of international medical graduates. Medical Journal of Australia. 2016;205(5):209-10.

[19] Australian Medical Council. AMC Accreditation of Workplace Based Assessment Providers: Standards and
Procedures. Kingston, ACT: Australian Medical Council; 2019.

[20] Elkin K, Spittal M, Studdert D. Risks of complaints and adverse disciplinary findings against international medical graduates in Victoria and Western Australia. Medical Journal of Australia. 2012;197(8):448-52.

[21] Malik A, Bhugra D. Workplace-based assessment methods: Literature overview. In: Malik A, Bhugra D, Brittlebank A, editors. Workplace-Based Assessments in Psychiatry. 2nd ed. London: RCPsych; 2011.

[22] Norman GR, Shannon SI. Effectiveness of instruction in critical appraisal (evidence-based medicine) skills: a critical appraisal. Can Med Assoc J. 1998;158(2):177-81.

[23] Booth J, Johnson G, Wade W. Workplace-Based Assessment Pilot: Report Findings of a Pilot Study. Royal College of Physicians; 2009.

[24] Miller A, Archer J. Impact of workplace based assessment on doctors' education and performance: A systematic review. Brit Med J. 2010;341:c5064.

[25] Nair BR, Alexander HG, McGrath BP, Parvathy MS, Kilsby EC, Wenzel J, et al. The mini clinical evaluation exercise (mini-CEX) for assessing clinical performance of international medical graduates. Medical Journal of Australia. 2008;189(3):159-61.

[26] Nair BR, Searles AM, Ling RI, Wein J, Ingham K. Workplace-based assessment for international medical graduates: at what cost? Med J Austalia. 2014;200(1):41-4.

[27] Jyothirmayi R. Case-based discussion: assessment tool or teaching aid? Clinical Oncology. 2012;24(10):649-53.

[28] Burford B, Rothwell C, Beyer F, Tanner L, Vance G. Best Practice in the Assessment of Competence: A Literature Review. 2018.

[29] Hatala R, Sawatsky AP, Dudek N, Ginsburg S, Cook DA. Using in-training evaluation report (ITER) qualitative comments to assess medical students and residents: a systematic review. Academic Medicine. 2017;92(6):868-79.

[30] Schifferdecker KE, Reed VA. Using mixed methods research in medical education: basic guidelines for researchers. Medical Education. 2009;43(7):637-44.

[31] Ivankova NV, Creswell JW, Stick SL. Using mixed-methods sequential explanatory design: From theory to practice. Field Method. 2006;18(1):3.

[32] Low, J. A Pragmatic Definition of the Concept of Theoretical Saturation. Sociological Focus. 2019;52(2):131-139.

[33] QRS International. Nvivo 10.0 Melbourne: QRS International Pty Ltd; 2012 [Available from: http://www.qsrinternational.com/\#tab_you.

[34] Braun V, Clarke V. Using thematic analysis in psychology. Qualitative Research in Psychology. 2006;3(2):77-101.

[35] O'Brien BC, Harris IB, Beckman TJ, Reed DA, Cook DA. Standards for Reporting Qualitative Research: A Synthesis of Recommendations. Academic Medicine. 2014;89(9).

[36] Pallant J. SPSS survival manual: a step by step guide to 
data analysis using SPSS for Windows. 4th ed. Crows Nest, N.S.W: Allen \& Unwin; 2011.

[37] Massie J, Ali JM. Workplace-based assessment: a review of user perceptions and strategies to address the identified shortcomings. Advances in Health Sciences Education. 2016;21(2):455-73.

[38] Shalhoub J, Vesey AT, Fitzgerald JEF. What evidence is there for the use of workplace-based assessment in surgical training? Journal of surgical education. 2014;71(6):906-15.

[39] Miller A, Archer J. Impact of workplace based assessment on doctors' education and performance: a systematic review. BMJ. 2010;341:c5064.

[40] McKavanagh P, Smyth A, Carragher A. Hospital consultants and workplace based assessments: how foundation doctors view these educational interactions? Postgraduate medical journal. 2012;88(1037):119-24.

[41] Bindal T, Wall D, Goodyear HM. Trainee doctors' views on workplace-based assessments: Are they just a tick box exercise? Medical teacher. 2011;33(11):919-27.

[42] Weller J, Jolly B, Misur M, Merry A, Jones A, Crossley JM, et al. Mini-clinical evaluation exercise in anaesthesia training. British Journal of Anaesthesia. 2009;102(5):633-41.

[43] Dean BJ, Duggleby PM. Foundation doctors' experience of their training: A questionnaire study. JRSM short reports. 2013;4(1):1-7.

[44] Basu I, Parvizi S, Chin K. The perception of online work based assessments. The clinical teacher. 2013;10(2):73-7. 